\title{
¿Qué tan competitivos son los países miembros de la Alianza del Pacífico en infraestructura de transporte? ${ }^{1}$
}

Raúl Alberto Cortés Villafradez ${ }^{2}$ Universidad Jorge Tadeo Lozano raul.cortesv@utadeo.edu.co

DOI: https://doi.org/10.21158/01208160.n85.2018.2055

Fecha de recepción: 06 de diciembre de 2017

Fecha de aprobación: 20 de marzo de 2018

Cómo citar este artículo / To reference this article / Comment citer cet article / Para citar este artigo:

Cortés Villafradez, R. A. (2018). ¿Qué tan competitivos son los países miembros de la Alianza del Pacífico en infraestructura de transporte? Revista EAN, 85, 143-162. DOI: https://doi.org/10.21158/01208160.n85.2018.2055

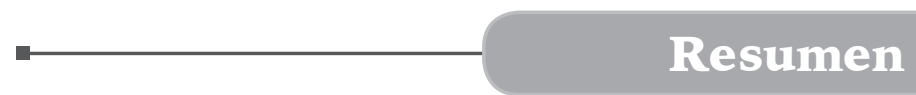

Este documento analiza por medio de un estudio descriptivo, cómo el desarrollo de infraestructura fue un elemento importante de la competitividad, durante el periodo 2006-2016. En esta investigación se compara el comportamiento de la inversión en infraestructura de transporte frente a variables que miden los resultados de la competitividad de la misma, como lo son: costos, tiempo, calidad y desempeño logístico para cada uno de los países miembros de la Alianza del Pacifico, basado en fuentes de información tales como el Banco Mundial, World Economic Forum (WEF), Economic Commission for Latin America and the Caribbean (ECLAC) e Infralatam. A partir de dicho análisis se pudo evidenciar la estrecha relación existente entre la calidad de la infraestructura de transporte y el nivel de competitividad de los países estudiados.

\section{Palabras clave}

Alianza Pacífico, infraestructura del transporte, competitividad, logística en los negocios, desempeño económico.

\footnotetext{
${ }^{1}$ Colaboradores: María Juliana Chacón Franco, Carlos Alexander Amaya Becerra, Lady Tatiana Liévano Rodríguez y Nicolás Rocancio. Estudiantes de la Facultad de Ciencias Económicas y Administrativas de la Universidad Jorge Tadeo Lozano, pertenecientes al Semillero de Investigación sobre Infraestructura y Competitividad.

2 Magíster en Gestión de Organizaciones de la Universidad EAN, especialista en Gerencia Financiera, Economista y Administrador de Empresas de la Pontificia Universidad Javeriana.
} 


\title{
How much competitive are those countries which are members of the Pacific Agreement in transportation infrastructure?
}

\begin{abstract}
This article aims at analyzing descriptively how the development of infrastructure is an important component of competitiveness in the period from 2006 to 2016. This paper compares the investment behavior in transportation infrastructure based on the variables which measure its competitive outcomes, among which costs, time, quality and logistic performance are found for each of the country members of the Pacific Agreement, taking into consideration information sources such as The World Bank, World Economic Forum (WEF), Economic Commission for Latin America and the Caribbean (ECLAC) and Infralatam. Based on this research study, it is shown that there is a close relationship between the quality of transportation infrastructure and the competitive level of the countries involved in such study.
\end{abstract}

Key words. Pacific Agreement, transportation infrastructure, competitiveness, business logistics, economic development.

\section{Dans quelle mesure les pays membres de l'Alliance Pacifique sont-ils compétitifs en matière d'infrastructures de transport?}

Résumé. Ce document analyse à travers une étude descriptive la manière dont le développement des infrastructures a été un pillier fondamental de la compétitivité sur la période 2006-2016. Cette étude compare le comportement de l'investissement en infrastructures de transport à des variables mesurant les résultats de compétitivité tels que les coûts, les délais, la qualité et la performance logistique des pays membres de l'Alliance Pacifique en s'appuyant sur des sources d'information fournies par la Banque mondiale, le Forum économique mondial (FEM), la Commission économique pour l'Amérique latine et les Caraïbes (CEPALC) et Infralatam. Grâce à cette analyse, il a été possible de démontrer le lien étroit existant entre la qualité des infrastructures de transport et le niveau de compétitivité des país étudiés.

Mots clefs. Alliance du Pacifique, infrastructure de transport, compétitivité, logistique commerciale, performance économique.

\section{Até que ponto os países membros da Aliança do Pacífico são competitivos em infraestrutura de transporte?}

Resumo. Este documento analisa por meio de um estudo descritivo, como o desenvolvimento de infraestrutura foi um elemento importante na competitividade, durante o período 2006-2016. Nesta pesquisa compara-se o comportamento do investimento em infraestrutura de transporte frente a variáveis que medem os resultados da competitividade da mesma, como são: custos, tempo, qualidade e desempenho logístico para a cada um dos países membros da Aliança do Pacifico, baseado em fontes de informação tais como o Banco Mundial, World Economic Fórum (WEF), Economic Commission for Latin America and the Caribbean (ECLAC) e Infralatam. A partir desta análise pôde-se evidenciar a estreita relação existente entre a qualidade da infraestrutura de transporte e o nível de competitividade dos países estudados.

Palavras-chave. Aliança Pacífico, infraestrutura do transporte, competitividade, logística nos negócios, desempenho econômico. 


\section{Introducción}

$\mathbf{C}^{1}$ objetivo de la presente investigación Lbusca analizar la relación existente entre la competitividad y la infraestructura de transporte de los países miembros de la Alianza del Pacífico (AP): Chile, Colombia, Perú y México, en el periodo 2006-2015.

En la primera parte, se efectuó un análisis conceptual y de referencia acerca de la competitividad e infraestructura, utilizando enfoques teóricos y análisis de hallazgos empíricos en estudios sobre la materia. En segundo lugar, se realizó un análisis de variables de infraestructura como la inversión y variables de competitividad, como los costos, tiempos, la calidad y el desempeño logístico. Finalmente, se concluyen los hallazgos de dicho estudio y se realizan recomendaciones con respecto al tema.

Este trabajo utiliza una investigación de tipo cualitativo de alcance descriptivo, en la que se realiza una revisión del estado del arte relacionado con el objeto de estudio y se utiliza para el análisis de bases de datos e información obtenida del Banco Mundial (BM), el World Economic Forum (WEF), Consejo Económico para América Latina (CEPAL) e Infralatam.

En el desarrollo de la investigación se pretende comprobar la hipótesis nula que vincula a la infraestructura como determinante del desarrollo de la competitividad de los países de la Alianza del Pacífico. Las preguntas de investigación son: ¿La inversión en infraestructura de transporte es una variable que determina el nivel de competitividad de los países miembros de la Alianza del Pacífico? ¿Cuáles son las principales variables de infraestructura del transporte que tienen mayor repercusión sobre la competitividad de los países de la Alianza del Pacífico?

\section{Marco teórico}

L a Alianza del Pacífico es una integración deconómica y de desarrollo entre Chile, Colombia, México y Perú, cuyo propósito es a través de la cooperación impulsar el desarrollo y la competitividad de los cuatros países miembros, «los miembros de la Alianza del Pacífico confían que esto es posible a través de un avance progresivo de la libre circulación de bienes, servicios, capitales y personas» (Alianza del Pacífico, s.f., p. 1.).

Para el mencionado desarrollo económico y para lograr una buena posición competitiva es muy importante contar con una infraestructura adecuada, cabe resaltar que la infraestructura abarca muchos sectores como el financiero, el de las telecomunicaciones, el energético, el de transporte, entre otros; este estudio se enfoca en la infraestructura de transporte, ya que se han evidenciado muchos cuellos de botella en este ámbito en la Alianza del Pacífico, los cuales son un limitante significativo de la competitividad.

Al revisar la literatura se ha podido identificar tres vertientes con las cuales se relaciona la infraestructura, la primera es con el crecimiento económico, la segunda con la competitividad

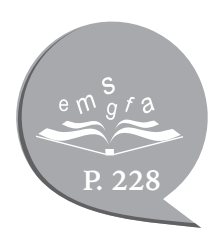


y por último se ha determinado que la competitividad es más compleja y que incluye diversos aspectos, entre estos la infraestructura.

\subsection{Infraestructura y crecimiento económico}

De acuerdo al estudio de la CEPAL (Consejo Económico para América Latina), Jaimurzina y Sánchez (2017) mencionan la importancia que tiene la gobernanza en infraestructura para lograr un desarrollo sostenible. Los hallazgos sugieren que es necesario un cambio en los servicios de infraestructura, ya que estos causan los principales desequilibrios en países de América Latina y el Caribe, los cuales se caracterizan por tener una producción poco diversificada, con escaza innovación, grandes desigualdades sociales y económicas y poca competitividad. Los resultados demuestran que un mejoramiento en aspectos de ingeniería e infraestructura física -energética, transporte, telecomunicaciones y agua potable- traen consigo externalidades positivas para toda la economía, mejorando como consecuencia la productividad, facilitando el comercio y permitiendo el desarrollo económico de un país o una región. Por lo tanto, una integración de todos los países en pro de este mejoramiento traería como resultado un desarrollo sostenible, el cual disminuiría la brecha con respecto a otros países y regiones.

Enfocándose en la infraestructura de transporte, Maza y Agámez (2012) han señalado que esta es una condición para el progreso y desarrollo económico, debido a que contribuye al buen desempeño de las unidades productivas, ya que influye en la estructura de costos, la productividad, la conectividad y la accesibilidad de las empresas, generando a su vez un impacto social. La disminución en los costos de transporte y el mejoramiento de las vías, de los puertos marítimos y de las condiciones del transporte fluvial son factores que influyen positivamente sobre la productividad y desarrollo.

Por lo que se puede afirmar la existencia de una relación directa entre la infraestructura de movilidad con el desarrollo económico, ya que es un aspecto relevante para potenciar una región; la mejora en la calidad de esta impacta positivamente en la competitividad empresarial, dando como resultado el posicionamiento de una región.

\subsection{Infraestructura y competitividad}

De acuerdo a Margot, Serebrisky, Suárez y Ramírez (2015), se encontró que la inversión pública y privada es importante para los países de América Latina para alcanzar un adecuado nivel de competitividad. En los últimos años países como Colombia, Ecuador, Chile, México, Brasil, Perú y Panamá han realizado inversiones estratégicas -ampliación de puertos marítimos, reformas de aeropuertos, concesiones viales, entre otros proyectos- los cuales han generado impactos positivos en el comercio exterior y la competitividad de dichos países.

De acuerdo con Castellanos, Lombana y Ortiz (2015), la primera apuesta para consolidar la competitividad de un país está asociada al desarrollo de una adecuada infraestructura y a la mejora de aspectos logísticos que permitan llevar sus mercancías al destino deseado, en el menor tiempo posible y al menor costo. Para los autores, la infraestructura del transporte en Colombia tiene una fuerte presión para su desarrollo y mejoramiento, derivada de la posición geográfica, de la firma de tratados de libre comercio y de acuerdos comerciales, los cuales aumentan significativamente el volumen de carga movilizada dentro del país. 
Sin embargo, según el Índice de Desempeño Logístico del Banco Mundial, Colombia desde el 2007, ha aumentado tres posiciones en América Latina, situándose en el puesto 12 entre 18 países, pero está siendo superado por más del $50 \%$ de los países del mundo, debido principalmente a una falta de infraestructura, calidad de los servicios de transporte e ineficiencia en aduanas y puertos (Consejo Privado de Competitividad, 2016).

Como se ha mencionado, Colombia presenta grandes rezagos en temas de infraestructura, lo que genera altos costos de producción, altos costos energéticos y altos costos de transporte de mercancía, mover un contenedor BogotáBuenaventura cuesta más de $\$ 1.000$ USD comparado con los precios en países de la Alianza el Pacífico; por lo que la Asociación Nacional de Instituciones Financieras (ANIF, 2017) ha creado un Índice de Competitividad Vial y Logística (ICVL), el cual analiza los diferentes pilares de la infraestructura vial, como lo son la infraestructura física, tecnológica, servicios logísticos y aspectos institucionales de los últimos 15 años en Colombia con respecto a la Alianza el Pacífico.

Se encontró que el número de carreteras pavimentadas, la calidad de la red vial, el número de peajes electrónicos, las inspecciones no intrusivas a consecuencia de la instalación de escáneres y la implementación de sistemas de georreferenciación y monitoreo en este sector son precarios, ineficientes y en algunos aspectos casi que inexistentes; sumado a esto se presentan altos costos al movilizar carga contenedorizada, altos tiempos en aduana y una baja inversión, que da como resultado una muy baja competitividad de Colombia frente a los países de la Alianza del Pacífico y en general a nivel mundial.
En un caso específico, Ángel (2010) estudia la infraestructura y competitividad de México. Su estudio concluye que la infraestructura es un punto clave en el mejoramiento competitivo del país. Se sugiere que para posicionar a México como una plataforma logística mundial es necesario un esfuerzo de todas las instituciones del país, y principalmente en la reducción de todos los costos y tiempos logísticos e impulsar el transporte intermodal del país. Por ende, se puede resaltar que la infraestructura en relación a la competitividad está estrechamente ligada al desarrollo de infraestructura logística en puertos, aeropuertos, red vial y férrea.

Estudios sugieren la importancia del desarrollo de la infraestructura como generador de externalidades positivas en la economía. Según García, Heredia y Pereira (2014), encuentran en su estudio que México ha logrado una posición competitiva gracias a las inversiones en infraestructura llevadas a cabo por parte del sector público a través de concesiones privadas. La importancia de sus inversiones en infraestructura de transporte radica en que le permitido mejorar la conectividad entre los centros productivos y centros logísticos aduaneros proporcionando reducciones en costos y en tiempos.

Adicionalmente, Sánchez y Wilmsmeier (2005) sugieren que Chile fue uno de los países que más invirtió en un cambio estructural para la mejora de la infraestructura de transporte, permitiéndole alcanzar mejoras en su competitividad. A nivel de Latinoamérica se encuentra posicionado como uno de los mejores países debido a sus bajos costos y ahorro de tiempos en operaciones logísticas relacionadas con el comercio internacional, ya que desde el año 1990 hasta el 2012, los gobiernos promovieron la vinculación de capital privado en un nivel del $26.6 \%$ del total de la 
inversión. Dichos recursos fueron destinados al equipamiento de infraestructura portuaria y proyectos viales, los cuales han generado una mejora en la calidad de prestaciones logísticas del país.

Perú ha logrado aumentar la tasa de crecimiento del PIB en un $8 \%$ gracias a las inversiones dirigidas a la mejora de la infraestructura de transporte y logísticas para dinamizar las actividades de exportación, lo que genera un aumento en la competitividad del país. De conformidad con Paredes (2012), se destaca el cambio que ha tenido Perú a lo largo de los últimos años, gracias a las inversiones públicas en proyectos dirigidos por el Centro Nacional de Planeamiento Estratégico (CEPLAN).

No obstante, existen problemáticas asociadas al desarrollo de la infraestructura, tanto en el acceso a mercados internacionales como a la competitividad de las economías. De acuerdo con Sánchez, Gallardo y Márquez (2011), en sus hallazgos sugieren que existe una precariedad en la infraestructura de transporte, lo cual ha dificultado el aprovechamiento de los acuerdos comerciales y los beneficios que aporta la globalización. Adicionalmente, Molina, Heuser y Mesquita (2016), sugieren que los mayores obstáculos al comercio que enfrenta actualmente la región radican principalmente en los costos derivados de una infraestructura de transporte, porque hacen que los beneficios del comercio lleguen solamente a un puñado de regiones y municipios ricos y bien conectados.

Finalmente, se requiere creación de instituciones y diseño de instrumentos detallados de política pública en materia de inversión en infraestructura de transporte por parte de los países para involucrar, regular y planear recursos para construcción de los proyectos de infraestructura que mejoren a su vez la competitividad. En este sentido, se requiere incrementar el nivel de inversión en infraestructura en una proporción superior al $50 \%$ del nivel actual (Barbero, 2011). Se requiere de un diseño de política pública apropiada para viabilizar proyectos de inversión de esta, que involucren combinaciones óptimas de fuentes de financiamiento público-privadas, definición clara de los riesgos inherentes a estos y las externalidades de inversión en la infraestructura necesaria para viabilizar las aspiraciones de crecimiento de la región Latinoamericana.

\subsection{Competitividad con base a diversos factores}

Por último, existe una vertiente la cual destaca que la competitividad de un país no depende solo de una variable, sino por el contrario demuestra que esta se relaciona con el buen desempeño de un grupo de variables en donde la infraestructura es importante.

El grado de competitividad de un país está dado por la capacidad de respuesta a los cambios del entorno, que es afectada positiva o negativamente por la situación económica, política, cultural y social del país; una economía es más competitiva mientras mayor sea la probabilidad de que crezca más rápido en el mediano o largo plazo. (World Economic Forum, 2010, p. 3)

Un estudio de Benzaquen, del Carpio, Zegarra y Valdivia (2010), quienes a partir del Informe de Competitividad Mundial del Foro Económico Mundial y del Anuario de Competitividad Mundial del Instituto Internacional para el Desarrollo de la Capacidad de Gestión, han diseñado un Índice de Competitividad Regional, donde se sustenta que los aumentos en la competitividad de un país están correlacionados con el crecimiento de la economía, por lo que depende de las acciones del Gobierno, 
del desarrollo económico, de una excelente infraestructura productiva y por último del capital humano.

Un análisis realizado para identificar la competitividad de la CAN (Comunidad Andina de Naciones), destacó que los factores que influyen en la competitividad son la infraestructura, la ciencia y tecnología, el mercado laboral y la productividad; a su vez se evidenció que la infraestructura es primordial, ya que permite desarrollar procesos productivos eficientes y sociales, por lo que se considera a este factor como el de mayor importancia en el ámbito competitivo e incluye un sinnúmero de aspectos que afectan su rendimiento, entre ellos carreteras y puertos, transporte aéreo, saneamiento y comunicación (Zapata, 2015).

Por otra parte, Álvarez (2011) ha comparado a Colombia frente al mundo teniendo en cuenta el índice de competitividad del Foro Económico Mundial, que se mide a través de tres subíndices, en los cuales se encuentran distribuidos 12 pilares que miden la competitividad.
Algunos de estos pilares incluyen, entre otros: infraestructura, entorno macroeconómico, salud y educación primaria, desarrollo de los mercados financieros, sofisticación de los negocios e innovación tecnológica.

Este índice posiciona a Colombia en el puesto 68 con 4.14 puntos en una escala de 1 a 7 , donde el país mejor posicionado es Suiza con 5.63 puntos, teniendo en cuenta los resultados, Colombia se encuentra en la mitad del índice, por lo que se considera que el país cuenta con una mala posición competitiva.

Desde cualquier vertiente que se analice se puede concluir que la infraestructura es primordial, ya que es capaz de potencializar las capacidades de una economía y por ende es una manera de responder a los retos que se imponen día a día en un país, una región y sobre todo aquellos que el mundo globalizado exige.
C on base en la literatura revisada, y teniendo en cuenta los datos e información recopilada a partir de 2008, se ha efectuado un análisis en materia de indicadores de infraestructura de transporte y competitividad, para cada uno de los países miembros de la Alianza del Pacífico.

\section{Análisis de la inversión en infraestructura}

Uno de los aspectos relevantes de dicho análisis fue el concerniente a los niveles de inversión que cada uno de los países miembros han realizado, en el sector de infraestructura, y en particular, en el subsector del transporte.

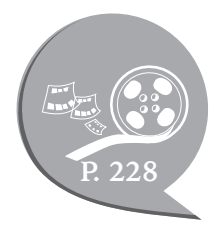


Figura 1. Inversión en infraestructura -todos los sectores-

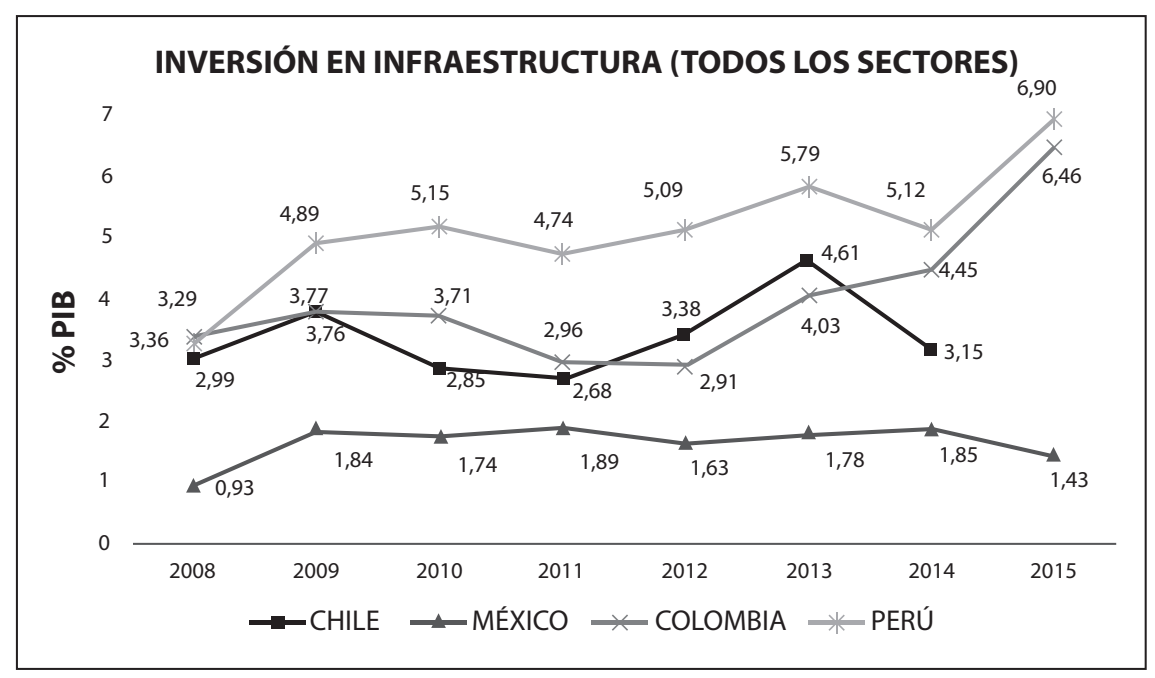

Fuente. Infralatam.

La inversión en infraestructura en materia de acueductos, energía, telecomunicaciones y transporte para los cuatro países miembros de la Alianza del Pacífico, presenta diferencias significativas, por lo cual no sigue una clara tendencia. Para el caso de Perú y Colombia, se puede evidenciar un comportamiento similar. Sin embargo, Perú es el país que a lo largo del tiempo ha destinado mayores recursos en infraestructura, con un promedio de $5.12 \%$ de su PIB. México por el contrario, fue el país que menos inversión realizó en dicho periodo, con un promedio de $1.64 \%$ de su PIB.

En año 2014, se registró una caída considerable de la inversión en infraestructura en Chile, con un $31.65 \%$, y en Perú con $11.57 \%$. En este sentido, dicha inversión en ambos países se vio afectada por la caída de la Inversión Extranjera Directa (IED) en $16 \%$ y $18 \%$ respectivamente. Caso contrario le sucedió a Colombia donde la inversión en infraestructura se incrementó junto con el aumento del $10 \%$ en la IED (CEPAL, 2014).

Tanto Colombia como Perú, presentaron variaciones importantes en materia de inversión en infraestructura para el periodo 2014-2015, con un incremento del 45.16 $\%$ para el primer país y del $34.76 \%$ para el segundo. Es importante mencionar, que aunque la inversión pública disminuyó, la privada aumentó en gran medida, estos dos países absorbieron gran parte de la IED en la Alianza del Pacífico.

En la figura 2 se puede evidenciar la inversión de los países miembros en infraestructura de transporte durante los años 2008 a 2015. 
Figura 2. Inversión en infraestructura de transporte

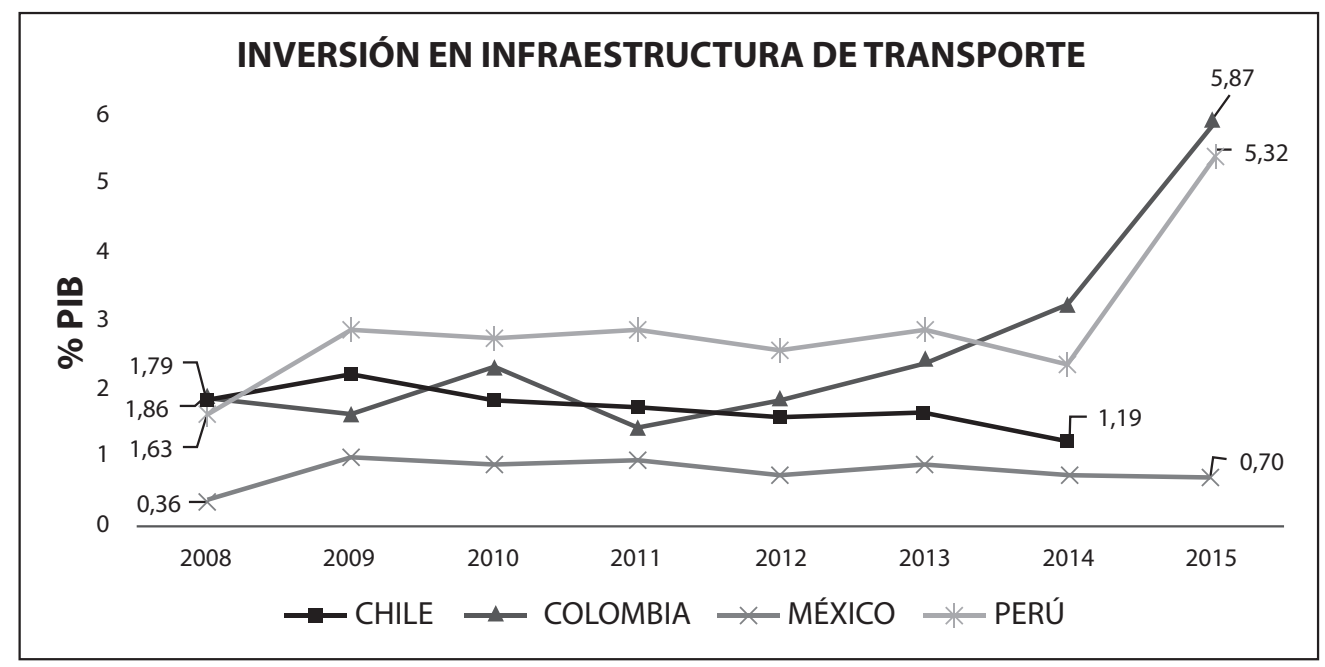

Fuente. Infralatam.

Se observa en las figuras 1 y 2 , que tanto la inversión en infraestructura de transporte como la inversión general de infraestructura, conservan la misma tendencia en su comportamiento.

Perú fue el país líder del grupo, con un promedio de $2.88 \%$ con relación a su PIB, seguido por Colombia con $2.56 \%$, Chile con $1.69 \%$, y finalmente, México con $0.77 \%$. Cabe resaltar que Colombia desde mediados del 2013, superó a Perú y se consolidó como el país que mayor porcentaje de su PIB destinó para este sector, el cual fue de $\$ 17.143,943$ millones de USD. Finalmente, México fue el país que menos recursos destinó a este sector, durante el periodo analizado.

\subsection{Calidad en la infraestructura}

Adicionalmente, en términos de calidad, se analizaron cuatro variables fundamentales para el desarrollo de infraestructura de transporte: carreteras, puertos, ferrocarriles y aeropuertos. Los datos fueron tomados de la Encuesta de Opinión Ejecutiva del World Economic Forum (WEF), aplicada a más de 14.000 líderes empresariales en 144 países, cuya calificación fue obtenida a partir de la respuesta de los encuestados a la calidad de infraestructura, donde 1 es subdesarrollada y 7 amplia y eficiente, según los estándares internacionales. 
Figura 3. Escala de calificación en calidad de carreteras y vías

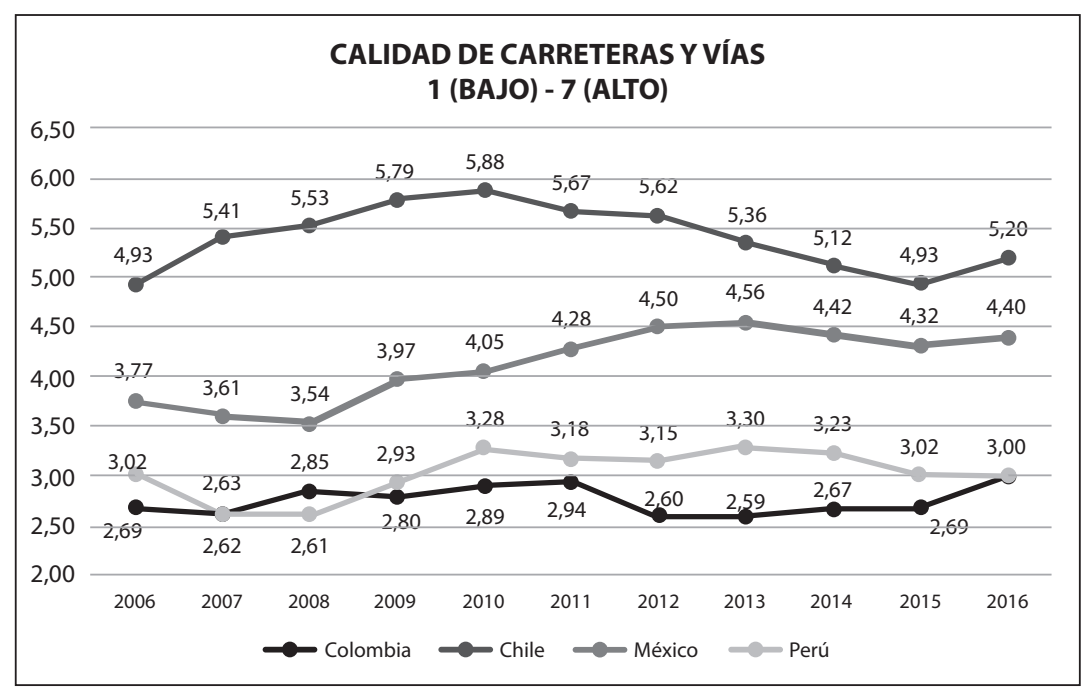

Fuente. The Global Economy - Foro Económico Mundial.

De acuerdo a los resultados obtenidos en la calidad de carreteras, el valor promedio para Colombia durante ese periodo fue de 2.76/7.0 puntos, con un mínimo de 2.69/7.0 en el año 2006 y un máximo de 3.0 registrado en el año 2016.

A su vez se observó que de los cuatro países Chile fue el país que presentó los mejores indicadores de calificación, su promedio fue de 5.40/7.0 puntos, constituyéndose como

Figura 4. Escala de calificación en calidad de puertos país líder de la Alianza del Pacífico en materia de calidad de carreteras y vías, su puntaje más alto fue de 5.88/7.0 en el año 2010. La calificación promedio para México fue de 4.13/7.0 y para Perú 3.03/7.0.

Se pudo determinar que el comportamiento de esta variable en cada país fue estable y presentó diferencias significativas entre los niveles de los países miembros.

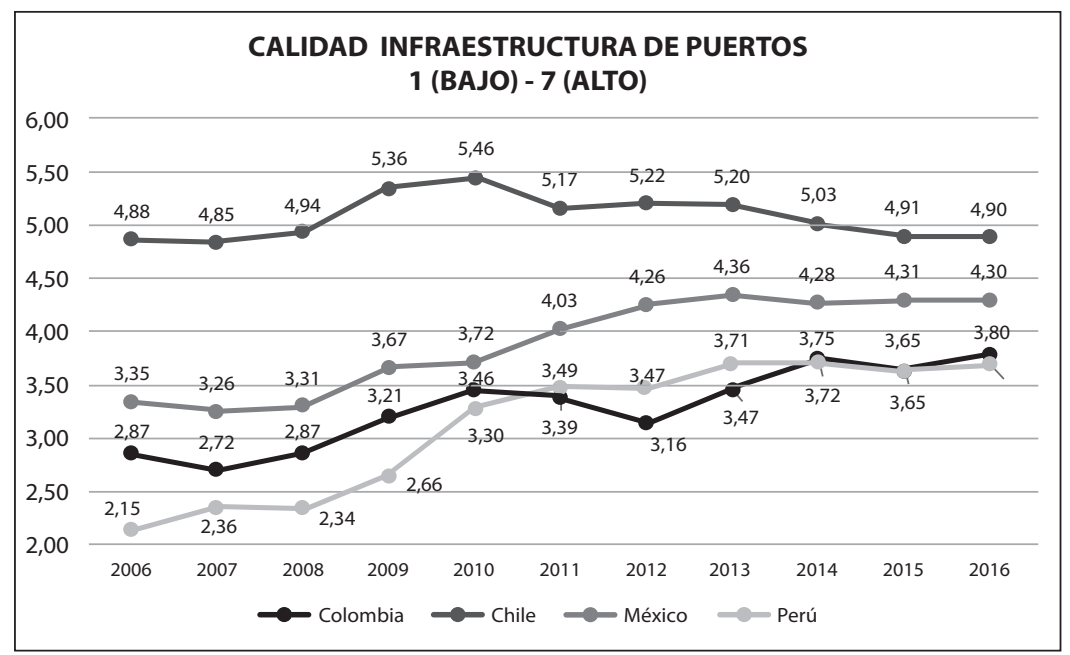

Fuente. The Global Economy - Foro Económico Mundial. 
Con relación al análisis de calidad infraestructura de puertos, se observó que Chile ha presentado una tendencia estable en sus calificaciones, alcanzando en el año 2010 un puntaje de 5.46/7.0, y un promedio de 5.08/7.0.

Cabe resaltar el mejoramiento progresivo que ha venido presentando Perú en este aspecto, y aunque en el año 2006 obtuvo un puntaje desfavorable de 2.15/7.0 puntos, logró recuperar su calidad de la infraestructura portuaria, lo cual se vio reflejado en el año 2016 con 3.70/7.0 puntos, con una media en su calificación 3.14/7.0 en el periodo 2006-2016.

México presentó una tendencia de crecimiento similar a la del Perú, con un promedio de 3.89/7.0. A pesar de que Colombia ha presentado una mejora en este indicador, aún tiene un importante rezago frente a México y Chile.

Figura 5. Escala de calificación en calidad de ferrocarriles

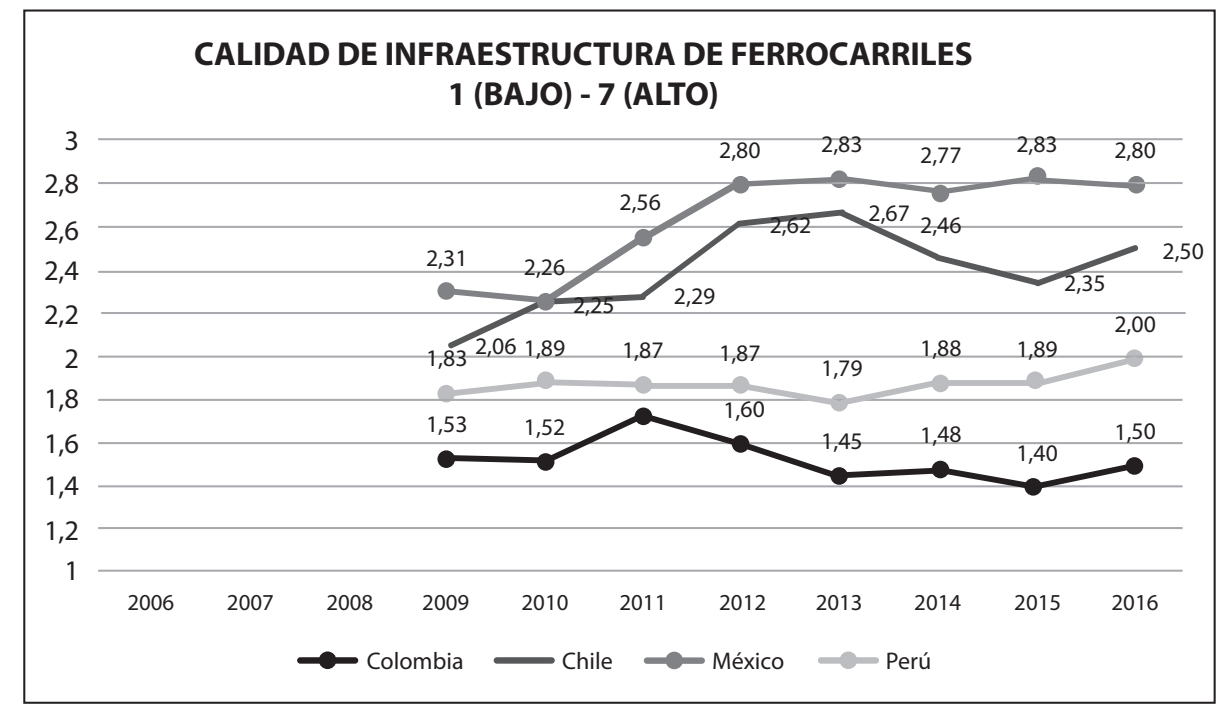

Fuente. The Global Economy - Foro Económico Mundial.

Para el indicador de calidad de infraestructura ferroviaria los datos disponibles se encontraron a partir del año 2009, dado que para los años anteriores no existen registros por parte del World Economic Forum (WEF).

En la figura 5 se observa que la calidad de la infraestructura de este medio de transporte en los países de la Alianza del Pacífico es precaria. No obstante, México es el país con la mejor calidad en sus ferrocarriles, mostrando un promedio de 2.65/7.0 puntos durante el periodo analizado, mientras que Colombia presentó el promedio más bajo, con 1.53/7.0, lo que nos revela un resultado en materia de calidad en el sistema ferroviario lamentable, producido por el deterioro en su calidad con el pasar de los años y por el descuido de la red ferroviaria por parte de las instituciones del Estado responsables de su manejo. 
Figura 6. Escala de calificación en calidad de transporte aéreo

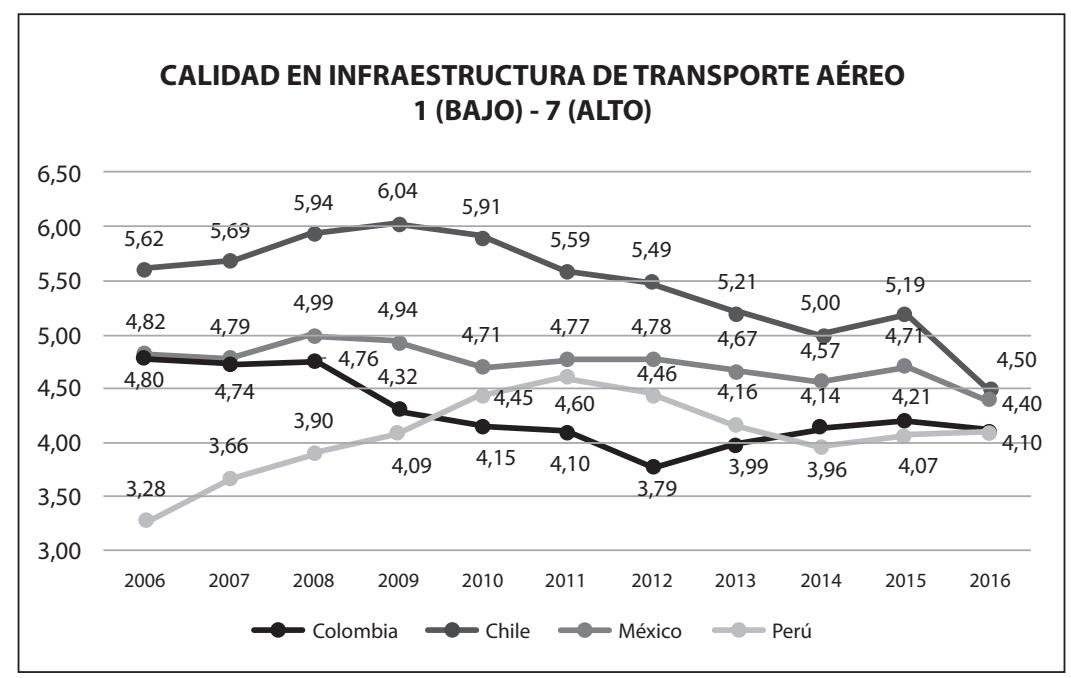

Fuente. The Global Economy - Foro Económico Mundial.

Por último, se analizó la calidad de infraestructura del transporte aéreo, evidenciando que Chile a pesar de ser el mejor calificado, viene presentando una tendencia decreciente bastante pronunciada, llegando a los 4.50/7.0 puntos en el 2016 con un promedio de 5.47/7.0.

Perú presentó un crecimiento de más de 1 punto en su calificación entre el 2006 hasta el 2011, alcanzando una calificación de 4.60/7.0, empero durante los últimos años este índice ha decaído. Colombia por su parte, desde año 2006 hasta el 2012 mostró una caída significativa, de la cual se ha venido recuperado a partir del año 2014, alcanzando el mismo puntaje que Perú para el 2016, siendo este de $4.10 / 7.0$ puntos.
Durante el periodo 2006-2016, México registró una caída del $8.7 \%$ en su calificación de calidad en infraestructura de transporte pasando de 4.8/7.0 a 4.4/7.0. No obstante, sigue posicionándose en el segundo lugar de la (AP) sobre la materia, después de Chile.

\subsection{Variables de competitividad}

\subsubsection{Análisis de costos y tiempos para exportar.}

Para hallar la relación existente entre la inversión en infraestructura y competitividad, es necesario examinar las variables que hacen parte del desarrollo competitivo de los países de la Alianza del Pacífico, por ende, se deben tener en cuenta factores de tiempos, costos y calidad, los cuales fueron analizados. 
Figura 7. Costo y tiempo para exportar a países Alianza del Pacífico

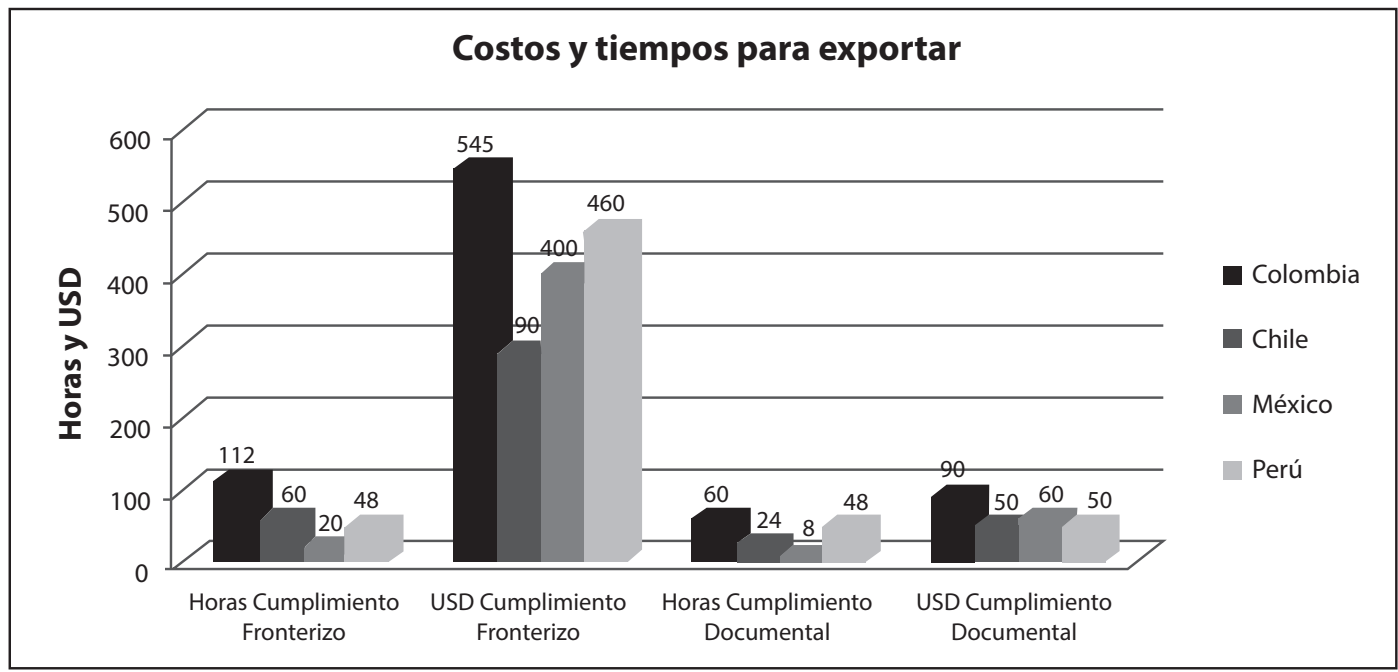

Fuente. Procolombia.

El costo y tiempo del cumplimiento fronterizo y documental para exportar incluye la obtención, preparación y presentación de documentos durante el manejo en puertos o fronteras, el despacho de aduanas y los procedimientos de inspección.

Para el caso de Colombia, registró el tiempo y el costo más elevado con respecto a los países de la referencia, con 112 horas y 545 USD, en el aspecto de cumplimiento fronterizo, seguido por Perú, el cual presentó tiempos de 48 horas y costos de 460 USD. Chile por su parte presentó 60 horas y los menores costos, reportados en 290 USD. Aunque México tan solo arrojó 20 horas, constituyéndose en el país de la Alianza del Pacífico con el menor tiempo para exportar, sus costos están por encima de los de Perú, siendo estos de 400 USD.

En temas de cumplimientos documentales los cuatro países presentan valores similares, cabe resaltar que Colombia es el país que ocupa la posición más desfavorable en tiempos y costos, mientras que México es el país con menores tiempos y Chile el país con menores costos. Los resultados favorables de Chile se deben a que desde hace muchos años el sector de transporte e infraestructura, ha sido de gran importancia, presentando una reducción de los costos y tiempos en la comercialización de mercancías, lo que le ha permitido mejorar su posición competitiva.

\subsubsection{Indicador de desempeño logístico.}

El Índice de Desempeño Logístico del Banco Mundial (IDL) nos muestra la calidad del comercio e infraestructura relacionada con el transporte de los países miembros de la Alianza del Pacífico para el 2007-2016.

Este índice tiene como fin medir la cadena logística de abastecimiento de los países, teniendo en cuenta factores como la aduana, infraestructura de transporte, envíos internacionales -facilidad de trámites-, calidad y competencia en logística, rastreo y seguimiento, y por ultimo puntualidad. 
Figura 8. Desempeño logístico de la calidad del comercio y la infraestructura del transporte

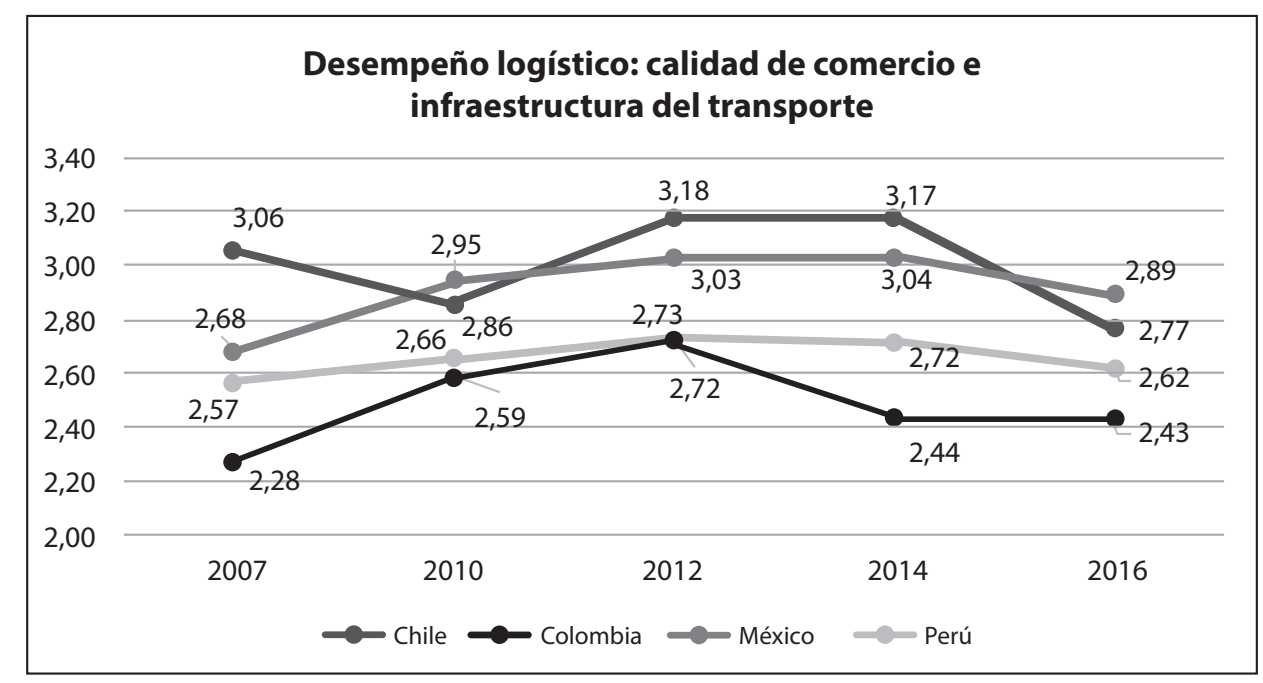

Fuente. Banco Mundial. Índices logísticos.

También fue analizado el comportamiento de la variable que refleja el grado de desarrollo de infraestructura sobre la competitividad, cuyo indicador es el Índice de Desempeño Logístico (IDL). Ese indicador es una variable compuesta que incluye el promedio de los puntajes de eficiencia de los procesos aduaneros -despacho-, la calidad de la infraestructura de transporte, la facilidad para realizar envíos internacionales y la competencia y calidad de servicios de logística, esta última contempla la eficiencia de los operadores de transporte y agentes de aduana, así como la capacidad de rastrear envíos y la puntualidad de estos en llegar a su destino final, cuya medición presenta una escala del 1 a 5 , siendo 5 la mejor ponderación; los datos corresponden al año 2016.

Figura 9. Desempeño logístico Alianza del Pacífico - 2016

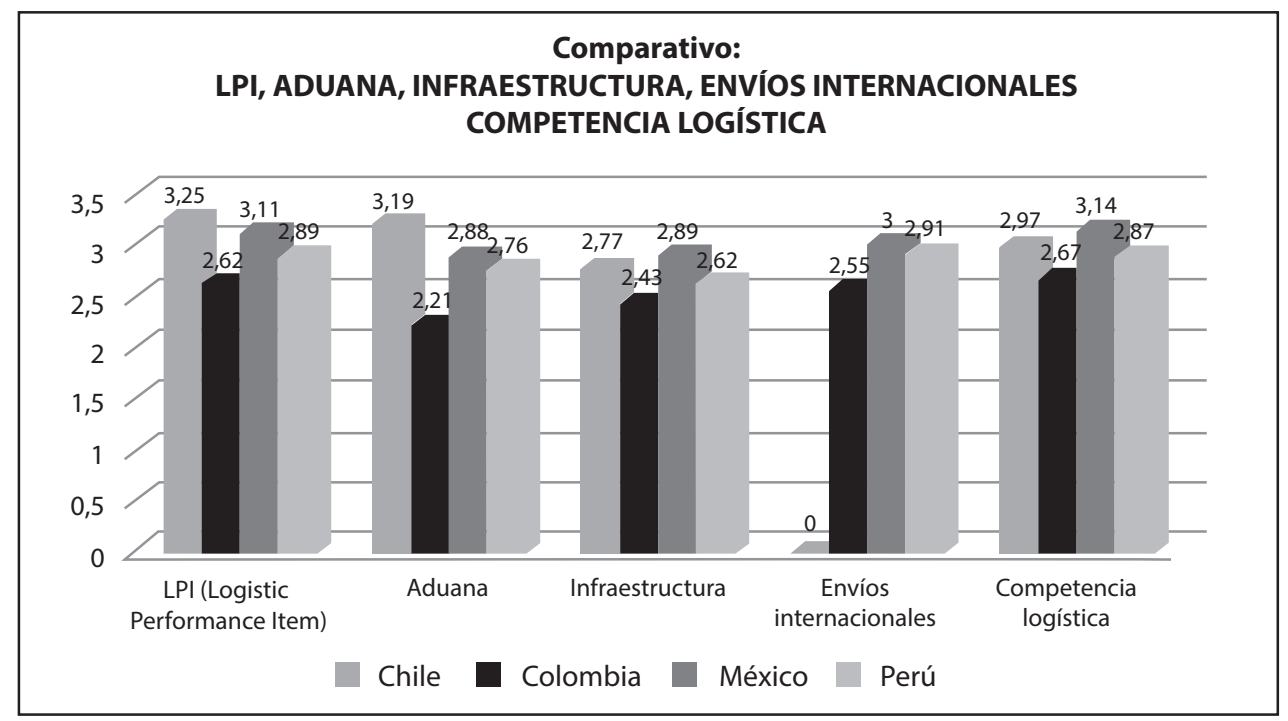

Fuente. World Bank. Datos del índice general LPI derivado. 
Mediante un análisis comparativo presentado en la figura 9, se ve reflejado el nivel de competitividad de los países miembros en temas de comercio exterior. En su última medición, los resultados sugieren que Chile y México son los países más competitivos de la Alianza del Pacífico en esta materia, los cuales desarrollan de manera más eficiente las variables que este índice mide, por tanto, se considera que sus procesos son más ágiles soportados en una calidad de infraestructura de transporte superior y métodos logísticos y aduaneros con un mayor grado de tecnificación con respecto a sus referentes.
Perú por su parte se encuentra muy cercano a los líderes debido a que sus diferencias son mínimas. Colombia muestra el peor desempeño en la Alianza del Pacífico a causa de sus complejos procesos y precaria infraestructura de transporte, principalmente aquella que va desde los centros productivos hasta las zonas de comercio exterior como puertos o aeropuertos, los cuales se traducen en mayores tiempos y costos, afectando directamente su nivel competitivo.

\section{Hallazgos}

\subsection{Caso de Colombia}

Históricamente, Colombia ha presentado atrasos en el desarrollo de su infraestructura general debido a la falta de inversión y a la débil planeación y ejecución de proyectos, entre otras razones. Bajo esta premisa se parte para determinar que hay un gran rezago en nivel de dotaciones de infraestructura, que influye notablemente en nuestro nivel de competitividad y en la participación de nuestra economía en el comercio internacional.

La calidad de las carreteras, los largos trayectos, el terreno montañoso y los altos costos que conlleva movilizar las mercancías o productos representan un gran impedimento en el desarrollo eficiente de nuestros procesos logísticos, afectando directamente nuestra transformación productiva, objetivo primordial de nuestra política de competitividad.

La liberalización comercial ha generado una mayor concentración económica en el centro del país, desarrollándose actividades como el "efecto costero", refiriéndose a la movilización de mercancías que se genera de la capital hacia los puertos. La concentración económica en las principales áreas metropolitanas es relativamente alta, sin embargo, y a pesar de la apertura económica, todavía hay regiones en Colombia que no se benefician del comercio internacional o del acceso a los mercados externos, debido a los altos costos del transporte interno y el difícil acceso a estas. La baja participación de algunas regiones como la Orinoquía, revelan claramente la problemática de infraestructura por la que se atraviesa: centros aduaneros alejados, cruce de cordilleras -lo que se traduce en transporte difícil y costoso-, vías secundarias y terciarias pocas o inexistentes e incluso el precio de los combustibles que interfiere en el desempeño óptimo de estas regiones en la participación de las exportaciones.

En las diferentes fichas bibliográficas analizadas, es posible evidenciar, por ejemplo, que una reducción en los costos de transporte interno en las regiones más apartadas de los puertos tendría una mejora significativa en el desempeño exportador de algunas regiones. En general, las regiones ubicadas en las áreas 
más retiradas de una aduana no solamente tienen costos de transporte elevados, sino que además sus niveles de exportación suelen ser menores, lo que sugiere que el desempeño comercial de las regiones remotas está limitado por la distancia y, en consecuencia, por los altos costos de transporte (Mesquita, 2013). Así mismo, las condiciones de las carreteras no son las mismas a lo largo del país, y mejorarlas podría generar un efecto particularmente importante en las regiones con un desempeño exportador inferior.

Los ejercicios de simulación confirman que el costo interno de transporte es una barrera que se interpone a la capacidad que tenga una región aislada de beneficiarse de las oportunidades del comercio internacional (Mesquita, 2013).

Así las cosas, es claro que en Colombia la red vial -ya sean primarias, secundarias o terciarias-, presenta muchos problemas: son cortas, angostas, rugosas etc., sumándose a esta problemática las condiciones geográficas de nuestro país -topografía-, lo que genera que la construcción de carreteras, túneles y demás obras de apoyo impliquen un mayor costo. Ahora bien, más allá de la calidad de las carreteras, puertos, ferrocarriles y aeropuertos, existen factores informales que generan altos costos en la contratación de los servicios de transporte, como lo es la industria de transporte de carga terrestre, donde además de no contar con camiones eficientes y apropiados para los diferentes tipos de carga, se tiene un control gubernamental sobre los precios del mismo.

\subsection{Caso de México}

Este panorama desalentador no solamente afecta a Colombia; México por su parte, aunque goza de una ubicación geográfica privilegiada -lo que constituye una ventaja logística importante- $\mathrm{y}$ su actividad productiva se beneficia de la vecindad del mercado de Estados Unidos, posee problemas similares de infraestructura de transporte. Aunque se considera que el $82 \%$ de la red carretera está en buenas condiciones, el diseño de su infraestructura que ha sido trazada directa hacia los Estados Unidos y los años de inversiones insuficientes han obstaculizado los planes del gobierno para abordar el acceso de las diferentes regiones al comercio, ocasionando disparidades territoriales. (Molina et al., 2016).

Adicionalmente, la inseguridad, los costos de los peajes y de combustible, seguros, licencias, permisos y el mantenimiento y sostenibilidad de sus medios de transporte en general son determinantes fundamentales de sus altos costos de transporte, afectando claramente sus indicadores de competitividad (IMCO, 2003).

A pesar de estas prominentes debilidades, México destina menos del $1 \%$ de su PIB en inversión para este sector.

No obstante, y considerando los diferentes indicadores de competitividad analizados por el WEF, México se encuentra relativamente mejor posicionado que Perú y Colombia en calidad de infraestructura de transporte, así como en desempeño logístico y eficiencia aduanera.

\subsection{Caso de Perú}

Perú, igualmente presenta retrasos significativos en temas de infraestructura de transporte que influyen en su crecimiento económico y competitividad. Similar al caso colombiano, su topografía es uno de los principales obstáculos, ya que posee uno de los terrenos más rugosos del mundo, sumado a su variabilidad en el clima, que genera que sus carreteras y diferentes redes de transporte sean menos densas y tiendan a deteriorarse con mayor facilidad (Mesquita, 
2013). Posee además, algunos obstáculos adicionales asociados a su desarrollo logístico como lo es la distancia de las aduanas, lo cual acentúa la disparidad regional. A pesar de esta realidad, el transporte por carretera es el más usado en el Perú, por lo que se han hecho grandes esfuerzos para lograr allegar sus productos exportables a los puntos de salida aprovechando su ventaja costera, catalogándose como uno de los países miembros de Alianza Pacífico que ha generado mayor inversión en su infraestructura de transporte durante los últimos años.

En cuanto a competitividad internacional, comparado con Colombia, Perú le aventaja en algunas de las variables incluidas en los determinantes de la competitividad -condiciones de factores, condiciones de demanda y estructura de las empresas- presentando mejores indicadores a nivel de crecimiento de exportaciones, inversión extranjera directa (IED), ingresos, tiempo para exportar $-36 \%$ menor que Colombia-, menor deuda pública, mayor valor agregado en servicios y exportación de metales y minerales (Castro, Vásquez y Vega, 2015).

Esto se puede evidenciar en los últimos reportes de competitividad publicados por el WEF, en el cual Perú desde el año 2010 viene mejorando su puntuación, presentando adelantos significativos, principalmente en el pilar de infraestructura. De igual manera, y de acuerdo a las diferentes cifras analizadas, el país Inca ha mostrado un crecimiento acelerado durante los últimos años, mostrando un desempeño exportador alto sustentado por una mayor inversión en su calidad de infraestructura de transporte, disminuyendo en cierto grado las brechas de su red de rutas, las cuales claramente no fueron construidas por razones asociadas al comercio internacional (Mesquita, 2013).

\subsection{Caso de Chile}

Finalmente, encontramos a Chile como el país miembro de Alianza Pacífico que presenta los mejores índices de competitividad derivado de la calidad superior de sus diferentes redes de transporte, apoyado en una estructura logística completa para sus operaciones de comercio exterior. Aunque también posee dificultades de concentración económica, tiene un sistema de producción por nodos, es decir que las industrias se concentran en zonas determinadas, así el acceso hacia estas, se realiza por medio de transporte vial, férreo o cabotaje, y permite que cada modo se especialice para el manejo de la carga (Legiscomex.com, 2013). El transporte férreo en Chile es un gran complemento del transporte carretero y marítimo, dado que transporta grandes volúmenes de carga, es más seguro para transportar sustancias o productos químicos, los tiempos de entrega son menores y tiene conexiones con grandes ciudades y centros de producción. Dentro de las operaciones de comercio exterior, el transporte marítimo es el modo más importante dado que moviliza $96.4 \%$ del total de importaciones y exportaciones de Chile (2012). El transporte aéreo moviliza solo el $1 \%$ del total de las operaciones de comercio exterior.

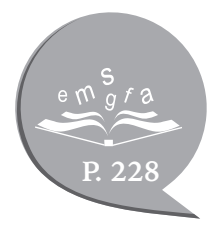




\section{Conclusiones y recomendaciones}

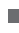

$\mathrm{D}_{\mathrm{r}}^{\mathrm{e}}$ acuerdo a los diferentes estudios realizados, basados en el comportamiento de las variables que miden el grado de desarrollo de la infraestructura de transporte junto con los indicadores de competitividad, se determina que existe una estrecha relación entre la calidad de infraestructura de transporte y el nivel de competitividad de los países.

Teniendo en cuenta la importancia que tienen los avances en materia de infraestructura de transporte, en la integración intrarregional de los países que componen la Alianza del Pacífico, se hace necesario desarrollar estudios posteriores en los que se analicen los impactos de la ejecución de dichos proyectos de inversión sobre el comercio -exportaciones e importaciones- tanto intrarregional como extraregional, como consecuencia de la reducción significativa en los costos y tiempos de transporte (Molina et al., 2016).

Ahora bien, esta relación para el caso de Perú y Colombia resulta nula, ya que aunque los planes de inversión han apuntado recientemente a la mejora de la infraestructura de transporte, existen otras variables relacionadas que intervienen en el desempeño competitivo, como lo son: costos de peajes, costos de combustible, costos financieros -depreciaciones-, licencias, permisos y demás. Es decir, mientras que no exista una regulación en materia de costos, peajes, tiempos, logística, entre otros aspectos, a pesar de destinar importantes montos de inversión infraestructura de transporte, esta no responderá de manera oportuna ni eficiente a las necesidades de los exportadores, ni contribuirá al mejoramiento de la competitividad.
Es decir que cuando se trata de reducir los costos de transporte no basta solo con construir carreteras; también es necesario diseñar políticas relacionadas con aspectos como la regulación, los incentivos y la estructura de mercado vigente (Mesquita, 2013).

Para el caso de Colombia se recomienda una relocalización de cadenas productivas, ya que gran parte de estas se encuentran localizadas a una distancia considerable de las principales zonas portuarias, ocasionando como consecuencia mayores costos que se ven representados en los fletes internos más altos de los cuatro países analizados.

Colombia ha presentado aumentos considerables en cuanto a la inversión en infraestructura vial, portuaria y aérea, sin embargo, su rezago con respecto a otros países de referencia es importante, lo que genera una posición desfavorable en el ranking mundial de competitividad. Las principales limitantes son: altos tiempo de entrega, mala calidad de las carreteras y poca conectividad entre sus puertos más importantes, altos costos de manejo de carga y complejidad en los trámites aduaneros, lo cual se traduce en aumento de los costos para el movimiento de mercancías.

Perú, al igual que Colombia, incrementó su inversión en el 2014, sin embargo, sus bajos niveles de productividad y competitividad son causados por la deficiente calidad y cantidad de la infraestructura vial. El principal destino de los recursos de infraestructura se direcciona al mejoramiento de sus puertos marítimos, lo que se ve reflejado en costos de fletes competitivos; y en menor medida al de los aeropuertos, lo cual ha generado un leve aumento de la productividad. 
En el caso de México se rechaza la hipótesis nula, toda vez que no existe una fuerte relación entre desarrollo de infraestructura con competitividad, por lo que se acepta la hipótesis alternativa. Lo anterior, teniendo en cuenta que México invierte un bajo porcentaje de su PIB a su infraestructura de transporte, en el índice general de competitividad, se encuentra mejor posicionado.

A pesar de que Chile y México poseen un stock de infraestructura de transporte más elevado, producto de las inversiones en proyectos, las cuales fueron realizadas en periodos previos al horizonte de estudio, y que se ven reflejadas en la calidad de sus carreteras, puertos y aeropuertos, se observó, sin embargo, que los flujos de inversión en los últimos años se redujeron por diversas causas como la devaluación de sus monedas y la caída de sus productos exportables.

\section{Referencias}

Alianza del Pacífico. (s.f.). ¿Qué es la Alianza? Recuperado de https://alianzapacifico.net/que-esla-alianza/\#la-alianza-del-pacifico-y-sus-objetivos

Álvarez, F. J. (2011). Índice de competitividad: Colombia frente al mundo. Lupa Empresarial, 30.

Ángel, R. J. (2010). Infraestructura y competitividad. En M. Barquin y F. J. Treviño (Coord..), La infraestructura pública en México: regulación y financiamiento (pp. 133-142). México D. F.: UNAM, Instituto de Investigaciones Jurídicas.

Anif. (2017). Índice de Competitividad Vial y Logística (ICVL) para Colombia. Bogotá.

Barbero, J. A. (2011). La infraestructura en el desarrollo integral para América Latina. Asunción: CAF.

Benzaquen, J., Del Carpio, L. A., Zegarra, L. A., y Valdivia, C. A. (2010). Un índice regional de competitividad para un país. Revista Cepal, 102, 6985.
Chile logra articular de manera más eficiente el transporte multimodal, por lo que se considera el país más competitivo de la Alianza del Pacífico.

En general en todos los países de la Alianza del Pacífico las inversiones en el sistema de redes ferroviarias han sido muy pobres y podrían constituirse en un medio de transporte mucho más eficiente, al poseer un menor costo fijo por tonelada de desplazamiento frente a otros medios de transporte.

Finalmente, se recomienda a todos los países miembros implementar instrumentos de política pública que permitan regular los sistemas de concesión de obras de infraestructura, sin afectar las variables de competitividad mencionadas anteriormente, así como promover un mayor dinamismo comercial y favorecer la eliminación de estos impedimentos u obstáculos.

Castellanos, A., Lombana, J. E., y Ortiz, M. (2015). Infraestructura logística y estrategias de marketing para la competitividad portuaria. Revista de Economía y Administración, 12(2), 95-112.

Castro, S., Vásquez, E., y Vega Vilca, J. (2015). Ecuador, Perú y Colombia: ¿Competidores o complementarios sudamericanos? Análisis de su competitividad global. Revista Global de Negocios, 1328. Recuperado de https://papers.ssrn.com/sol3/ papers.cfm?abstract_id $=2658854$

CEPAL. (24 de octubre de 2014). Disminuye en $23 \%$ inversión extranjera directa en América Latina en el primer semestre de 2014. Recuperado de https://www.cepal. org/es/comunicados/disminuye-en-23-inversionextranjera-directa-en-america-latina-en-el-primersemestre-de

Consejo Privado de Competitividad. (2016). Desempeño logístico: infraestructura, transporte y logística. Bogotá. 
García, J., Heredia, W., y Pereira, P. (2014). La Alianza del Pacifico. Una nueva era para América Latina. Ciudad de México: PwC México.

IMCO. (2003, septiembre). Análisis de la competitividad de México. Instituto Mexicano para la Competitividad. Recuperado de http://www.joseacontreras. net/mexico/economia/imco/imco_Pdfs/ analcompetitividad/archivoPublicacion(6) AnalCompetMex.pdf

Infralatam. (s.f.). Datos de inversión en infraestructura económica. América Latina y el Caribe.

Jaimurzina, A., y Sánchez, R. (2017). Gobernanza de la infraestructura para el desarrollo sostenible en América Latina y el Caribe: una apuesta inicial. Boletín FAL, (354), 1-14.

Legiscomex.com. (14 de agosto de 2013). Infraestructura logística en Chile y sus perspectivas. Recuperado de https://www.legiscomex.com/BancoMedios/ Documentos\%20PDF/estudio-logistica-chilerci-277.pdf

Margot, D., Serebrisky, T., Suárez, A., y Ramírez, M. C. (2015). Financiamiento de la infraestructura en América Latina y el Caribe: ¿Cómo, cuánto y quién? Washington D. C., USA: Banco Interamericano de Desarrollo.

Maza, F. J., y Agámez, A. D. (2012). La infraestructura de movilidad y su relación con el desarrollo económico y la competitividad. Panorama Económico, (20), 147-164.
Mesquita, M. (2013). Muylejos para exportar. Washington D. C.: Banco Interamericano de Desarrollo.

Molina, D., Heuser, C., y Mesquita, M. (2016). Infraestructura y desempeño de las exportaciones en la Alianza del Pacífico. Washington: BID.

Paredes, C. (2012). Plan estratégico sectorial multianual, sector transportes y comunicaciones 2012-2016. Lima: Oficina General de Planeamiento y Presupuesto.

Sánchez, D. M., Gallardo, D. L., y Márquez, P. N. (2011). ¿La infraestructura vial colombiana impulsa la competitividad del país? Bogotá: Universidad Militar Nueva Granada.

Sánchez, R., y Wilmsmeier, G. (2005). Provisión de infraestructura de transporte en América Latina: experiencia reciente y problemas observados. Santiago de Chile: CEPAL, Naciones Unidas.

World Economic Forum. (2010). Competitiveness Report 2010-2011. Génova, Suiza.

Zapata, C. V. (2015). Análisis de competitividad de los países de la Comunidad Andina de Naciones. Revista de Economía \& Administración, 12(1), 57-83. 